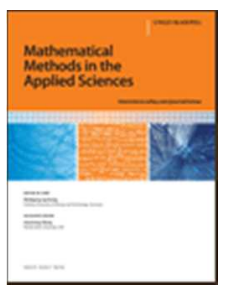

\title{
Miniversal deformations of observable marked matrices
}

\begin{tabular}{|r|l|}
\hline Journal: & Mathematical Methods in Applied Sciences \\
\hline Manuscript ID: & Draft \\
\hline Wiley - Manuscript type: & Paper \\
\hline Date Submitted by the Author: & n/a \\
\hline Complete List of Authors: & $\begin{array}{l}\text { Compta, Albert; Universitat Politecnica de Catalunya, } \\
\text { Ferrer, Josep; Universitat Politecnica de Catalunya, } \\
\text { Peña, Marta; Universitat Politecnica de Catalunya, ; }\end{array}$ \\
\hline Keyword: & $\begin{array}{l}\text { vertical pairs of matrices, conditined invariant subspaces, marked } \\
\text { subspaces, miniversal deformations }\end{array}$ \\
\hline
\end{tabular}




\title{
Miniversal deformations of observable marked matrices
}

\author{
Albert Compta, Josep Ferrer, Marta Peña ${ }^{1}$ \\ Departament de Matemàtica Aplicada I \\ Escola Tècnica Superior d'Enginyeria Industrial de Barcelona \\ Universitat Politècnica de Catalunya \\ Diagonal 647, 08028 Barcelona, Spain \\ E-mails: \{albert.compta,josep.ferrer,marta.penya\}@upc.edu
}

Keywords: Conditioned invariant subspaces, Miniversal deformation, Stratified manifold, Vertical pairs of matrices.

\begin{abstract}
Given the set of vertical pairs of matrices $\mathcal{M} \subset M_{m, n}(\mathbb{C}) \times M_{n}(\mathbb{C})$ keeping the subspace $\mathbb{C}^{d} \times\{0\} \subset \mathbb{C}^{n}$ invariant, we compute miniversal deformations of a given pair when it is observable and the subspace $\mathbb{C}^{d} \times\{0\}$ is marked. Moreover, we obtain the dimension of the orbit, characterize the structurally stable vertical pairs and study the effect of each deformation parameter.
\end{abstract}

\section{Introduction}

This paper contributes to the study of versal deformations when square matrices or pairs of matrices are considered, together with invariant subspaces. Versal deformations were introduced by Arnold in [1] (see also [2]) to study the variations of the invariants of a square matrix when its entries are perturbed. Thanks to a natural generalization contained in [22], the same technique has been applied to other cases, such as perturbations of pairs of matrices representing linear systems $([12],[13])$.

Invariant subspaces play a key role both in matrix theory (see [18]) and linear control systems (see [24]), where they are often called "conditioned" invariant subspaces. For instance, in [24] invariant subspaces are used in control problems such as Disturbance Decoupling or Output Regulation. From a theoretical point of view, the differentiable structure of the set of invariant subspaces of a square matrix has been studied in [21] and that of conditioned invariant subspaces of a pair in [16] and [17].

\footnotetext{
${ }^{1}$ Supported by DGICYT MTM2011-23892.
} 
In the context of versal deformations, it seems natural to consider the situation when both a matrix and an invariant subspace are involved and both or one of the elements of this couple is perturbed. So, in [10] the perturbation of a square matrix preserving an invariant subspace is studied. In particular, this perturbation gives all the solutions of the Carlson problem, and hence explicit realizations can be obtained (see [7]).

Here we complete the generalization of [10] to linear systems started in [9]. That is to say, we consider the perturbation of a pair of matrices preserving a given conditioned invariant subspace: we obtain the explicit form of the miniversal deformation of a given pair when it is observable and the subspace is marked (that is to say, when there is a BK-basis of the subspace extendible to a BK-basis of the whole space), by solving the equations obtained in [9]. Some applications are derived: computation of the dimension of the orbits, characterization of structurally stable objects, study of bifurcations diagrams...

We use the following notation. We write $M_{p, q}(\mathbb{C})$ the set of complex matrices having $p$ rows and $q$ columns. If $p=q$, we simply write $M_{p}(\mathbb{C})$, and $G l(p)$ will be the group of non-singular matrices in it. We consider the set $\mathcal{M} \subset M_{n+m, n}(\mathbb{C})$ of vertically embedded pairs of matrices having $\mathbb{C}^{d}$ as a conditioned invariant subspace. We restrict ourselves to the maximal stratum $\mathcal{M}^{*}$, which is an open dense set in $\mathcal{M}$.

The organization of this paper is as follows. In Section 2 and Section 3 we recall some results obtained in [9] regarding the differentiable structure of $\mathcal{M}$ and the implicit form of a miniversal deformation of a pair $\mathcal{A} \in \mathcal{M}^{*}$, respectively. In Section 4, we solve the above equations when the pair is observable and the subspace is marked (Theorem 16), also obtaining a second miniversal deformation without repeated parameters (Theorem 18). Finally, in Section 5 we obtain the dimension of the orbit (Corollary 19), characterize the structurally stable vertical pairs (Corollary 21) and study the effect of each deformation parameter.

\section{Pairs of matrices having a $(C, A)$-invariant fixed subspace}

We will deal with matrices of the form $\left(\begin{array}{c}A \\ C\end{array}\right) \in M_{n+m, n}(\mathbb{C})$, which we will identify with the pairs of matrices $(C, A)$, and which will be simply denoted as $\mathcal{A}$ if no confusion is possible.

Definition 1 Given a pair of matrices $(C, A) \in M_{n+m, n}(\mathbb{C})$, the pair $(C, A)$ is 
called observable if

$$
\operatorname{rank}\left(\begin{array}{c}
C \\
C A \\
\vdots \\
C A^{n-1}
\end{array}\right)=n
$$

We recall the definition of BK-equivalence or block similarity between pairs of matrices:

Definition 2 Given two pairs of matrices $(C, A),\left(C^{\prime}, A^{\prime}\right) \in M_{n+m, n}(\mathbb{C})$, we say that they are Brunovsky-Kronecker equivalent (or simply BK-equivalent) or blocksimilar if there are matrices $P \in G l(n), Q \in G l(m)$ and $R \in M_{n, m}(\mathbb{C})$ such that

$$
A^{\prime}=P A P^{-1}+R C P^{-1}, \quad C^{\prime}=Q C P^{-1} .
$$

The above pairs of matrices can be reduced to the following form:

Theorem 3 [14] Given $(C, A) \in M_{n+m, n}(\mathbb{C})$, there exist integers $k_{1} \geq k_{2} \geq \cdots \geq$ $k_{s}>0$ called Brunovsky-Kronecker indices (or simply BK-indices) and convenient matrices $P, Q, R$ as in Definition 2 which transform $(C, A)$ in its hereafter called BK-canonical form

$$
\mathcal{A}_{B K}=\left(\begin{array}{cc}
N & 0 \\
0 & J \\
\hline E & 0 \\
0 & 0
\end{array}\right)
$$

where

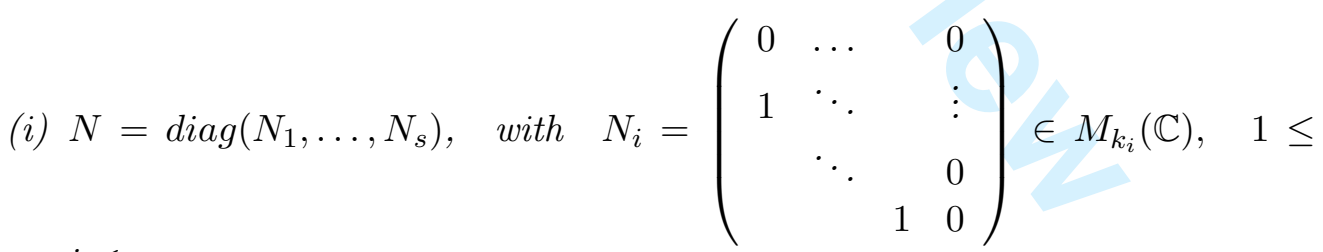
$i \leq s$,

(ii) $E=\operatorname{diag}\left(E_{1}, \ldots, E_{s}\right)$, with $E_{i}=\left(\begin{array}{llll}0 & \cdots & 0 & 1\end{array}\right) \in M_{1, k_{i}}(\mathbb{C}), \quad 1 \leq$ $i \leq s$,

(iii) $J$ is a Jordan matrix.

We refer the matrices of the above form as a BK-matrix. If $s=m$ it is said that it has full rank.

The block $J$ does not appear if $(C, A)$ is observable. 
We are interested in pairs $(C, A)$ having a fixed subspace $S \in \mathbb{C}^{n}$ as "invariant", in the sense of [3].

Definition $4 A$ subspace $S \subset \mathbb{C}^{n}$ is $(C, A)$-invariant or conditioned invariant if $A(S \cap \operatorname{Ker} C) \subset S$.

When conditioned invariant subspaces are involved, the BK-equivalence in Definition 2 is restricted in a natural way:

Definition 5 Given two pairs of matrices $(C, A),\left(C^{\prime}, A^{\prime}\right) \in M_{n+m, n}(\mathbb{C})$ having $S \subset \mathbb{C}^{n}$ as a conditioned invariant subspace, we say that they are $S$-equivalent (or simply equivalent if no confusion is possible) if

(i) the pairs are BK-equivalent, that is, $A^{\prime}=P A P^{-1}+R C P^{-1}, C^{\prime}=Q C P^{-1}$, where $P \in G l(n), Q \in G l(m), R \in M_{n, m}(\mathbb{C})$, and

(ii) the change of basis $P$ preserves $S$, that is, $P S=S$.

The subspace $S$ may be identified with $\mathbb{C}^{d}$ by considering "adapted basis":

Definition 6 Let us consider a subspace $S \subset \mathbb{C}^{n}, \operatorname{dim} S=d$. A basis of $\mathbb{C}^{n}$ whose $d$ first vectors form a basis of $S$ is called an adapted basis to the subspace.

In the above conditions, we will assume that the matrices $A$ and $C$ are blockpartitioned into

$$
A=\left(\begin{array}{cc}
A_{1} & A_{3} \\
A_{4} & A_{2}
\end{array}\right), \quad C=\left(\begin{array}{cc}
C_{1} & C_{2}
\end{array}\right),
$$

where $A_{1} \in M_{d}(\mathbb{C}), C_{1} \in M_{m, d}(\mathbb{C})$, and the matrix of the basis change $P$ into

$$
P=\left(\begin{array}{cc}
P_{1} & P_{3} \\
0 & P_{2}
\end{array}\right)
$$

where $P_{1} \in M_{d}(\mathbb{C})$, and the left bottom block being zero is just the condition $P S=S$.

Now we define and characterize the set $\mathcal{M}$ formed by the pairs of matrices having the subspace $S \subset \mathbb{C}^{n}$ as a conditioned invariant:

Definition 7 Given $S=\mathbb{C}^{d} \subset \mathbb{C}^{n}$, let

$$
\mathcal{M}=\left\{(C, A) \in M_{n+m, n}(\mathbb{C}): A(S \cap \operatorname{Ker} C) \subset S\right\}
$$

(that is to say, $S$ is $(C, A)$-invariant.)

Moreover,

$$
\mathcal{M}^{*}=\left\{\mathcal{A} \in \mathcal{M}: \operatorname{rank} C_{1}=r^{*}\right\},
$$

where $r^{*}=\min (d, m)$. 
In [9] one characterizes (Proposition 8[9]) the elements of the set $\mathcal{M}$. Moreover, one shows (Theorem 10[9]) that these elements form a stratified manifold and that the stratum $\mathcal{M}^{*}$ is an open dense subset in $\mathcal{M}$. Finally one describes (Proposition 12[9]) a Lie group that acts on $\mathcal{M}$ in such a way that the orbits are just the equivalence classes in Definition 5.

\section{Miniversal deformation preserving a (C,A)- invariant subspace}

In order to study the perturbations of a pair $(C, A)$ preserving a conditioned invariant subspace, we will use Arnold's techniques of the so-called versal deformations (that is, canonical forms of local differentiable families of perturbations). The starting point is the fact that the equivalence classes are orbits under the action of a Lie group, and hence they are submanifolds.

Then versal/miniversal deformations can be obtained as submanifolds which are transversal/minitransversal to the orbit. We recall some general definitions and results which we will apply to $\mathcal{N}=\mathcal{M}^{*}$.

Definition 8 Let $\mathcal{N}$ be a manifold. An l-dimensional deformation of $\mathcal{A} \in \mathcal{N}$ is a differentiable map

$$
\varphi: \Lambda \longrightarrow \mathcal{N}
$$

where $\Lambda$ is a neighborhood of the origin in $\mathbb{C}^{l}$ and $\varphi(0)=\mathcal{A}$. The image $\varphi(\Lambda)$ is said to be a family of deformations of $\mathcal{A} \in \mathcal{N}$.

If there is a Lie group $\mathcal{G}$ acting on the differentiable manifold $\mathcal{N}, \mathcal{G} \times \mathcal{N} \longrightarrow \mathcal{N}$, $(\mathcal{P}, \mathcal{A}) \longmapsto \mathcal{P} * \mathcal{A}$, a deformation is called "versal" if any other deformation is induced from it in the following sense:

Definition 9 Let $\mathcal{N}$ be a manifold and $\mathcal{G}$ a Lie group acting on it. A deformation of $\mathcal{A} \in \mathcal{N}, \varphi: \Lambda \longrightarrow \mathcal{N}$ is called versal if, given any other deformation of $\mathcal{A} \in \mathcal{N}$, $\psi: \Gamma \longrightarrow \mathcal{N}$, there is a neighborhood of the origin $\Gamma^{\prime} \subset \Gamma$, a differentiable map $\rho: \Gamma^{\prime} \longrightarrow \Lambda$ and a deformation of the identity $I \in \mathcal{G}, \delta: \Gamma^{\prime} \longrightarrow \mathcal{G}$ such that

$$
\psi(\tau)=\delta(\tau) * \varphi(\rho(\tau)), \forall \tau \in \Gamma^{\prime}
$$

It is called miniversal if it has the minimal dimension among the versal deformations.

Remark 10 In order to get a miniversal deformation of a point it is enough to compute it at any other point of the orbit: then the desired one is induced by the group action. 
We now recall the key relation between "versality" and "transversality" proved in [1] for square matrices, and which can be generalized (for example [22]) to the cases like the above one, where the equivalence classes are submanifolds given as orbits under the action of a Lie group.

Theorem 11 In the above conditions, (any parameterization of) the submanifold $\mathcal{L} \subset \mathcal{N}$ is a versal deformation of $\mathcal{A} \in \mathcal{L}$ if and only if $\mathcal{L}$ is transversal to $\mathcal{O}_{\mathcal{A}}$ at $\mathcal{A}$, that is to say, if

$$
T_{\mathcal{A}} \mathcal{L}+T_{\mathcal{A}} \mathcal{O}_{\mathcal{A}}=T_{\mathcal{A}} \mathcal{N}
$$

where $T_{\mathcal{A}}(\cdot)$ means the tangent space to $(\cdot)$ at point $\mathcal{A}$. Furthermore, it is miniversal if and only if it is minitransversal, that is to say, if the above sum is direct. Notice that, if $\mathcal{L}$ is a miniversal deformation of $\mathcal{A} \in \mathcal{N}$, then

$$
\operatorname{dim} \mathcal{O}_{\mathcal{A}}=\operatorname{dim} \mathcal{N}-\operatorname{dim} \mathcal{L}
$$

Thus, applying these techniques, one obtains the main result in [9], valid for a generic pair $\mathcal{A} \in \mathcal{M}^{*} \subset \mathcal{M}$ :

Theorem 12 [9] Let $\mathcal{A}=(C, A) \in M_{n+m, n}(\mathbb{C})$ be a pair of matrices and $S \subset \mathbb{C}^{n}$ a d-dimensional $(C, A)$-invariant subspace:

$$
\mathcal{A}=\left(\begin{array}{c}
A \\
C
\end{array}\right)=\left(\begin{array}{cc}
A_{1} & A_{3} \\
0 & A_{2} \\
\hline C_{1} & C_{2}
\end{array}\right)
$$

Assume $\mathcal{A} \in \mathcal{M}^{*}$, that is to say, $\operatorname{rank} C_{1}=r^{*}=\min (d, m)$. Then the following statements hold.

(i) If $m \leq d$, a miniversal deformation of $\mathcal{A}$ in $\mathcal{M}^{*}$ preserving $S$ as conditioned invariant subspace is given by the linear submanifold $\mathcal{L} \subset \mathcal{M}^{*}$ formed by the matrices:

$$
\mathcal{A}+\left(\begin{array}{cc}
X_{1} & X_{3} \\
Z\left(C_{1}+Y_{1}\right) & X_{2} \\
\hline Y_{1} & Y_{2}
\end{array}\right)
$$

satisfying the conditions

(1) $X_{2} C_{2}^{*}+Z=0$,

(2) $Y_{1} C_{1}^{*}+Y_{2} C_{2}^{*}=0$,

(3) $X_{1} C_{1}^{*}+X_{3} C_{2}^{*}=0$,

(4) $X_{3} A_{2}^{*}-A_{1}^{*} X_{3}-C_{1}^{*} Y_{2}=0$,

(5) $X_{1} A_{1}^{*}+X_{3} A_{3}^{*}-A_{1}^{*} X_{1}-C_{1}^{*} Y_{1}=0$,

(6) $X_{2} A_{2}^{*}-A_{3}^{*} X_{3}-A_{2}^{*} X_{2}-C_{2}^{*} Y_{2}=0$. 
(ii) If $d<m$, without loss of generality we can assume $C_{1}^{t}=\left(I_{d}, 0\right)$. Then, a miniversal deformation of $\mathcal{A}$ in $\mathcal{M}^{*}$ preserving $S$ as conditioned invariant subspace is given by the set of matrices

$$
\mathcal{A}+\left(\begin{array}{cc}
X_{1} & X_{3} \\
Z\left(I_{d}+Y_{11}\right) & X_{2} \\
\hline Y_{1} & Y_{2}
\end{array}\right),
$$

where $Y_{1}=\left(\begin{array}{c}Y_{11} \\ Y_{12}\end{array}\right), \quad Y_{11} \in M_{d}(\mathbb{C})$, satisfying (2)-(6) above and

$\left(1^{\prime}\right) \quad X_{2} C_{2}^{*}+(Z, 0)=0$, with $Z \in M_{n-d, d}(\mathbb{C})$,

\section{Miniversal deformations of observable marked matrices}

In [18] the "interesting class" of the so-called marked subspaces, namely, the invariant subspaces having a Jordan basis which can be extended to a Jordan basis of the whole space, is introduced. For instance, in [6] one proves that the "simplest" solutions of the Carlson problem are marked, and any other appears in a neighborhood of the marked ones. This notion was extended to pairs of matrices in [5] and used in [7] for the analogue to the Carlson problem: again the marked solutions cover all the possibilities and are the simplest realizations. Moreover, from the versal deformation of a pair in [12] it follows that "minimal" observable perturbations of a non-observable pair are marked.

Generalizing the concept of a marked subspace with regard to an endomorphism, we say that a $(C, A)$-invariant subspace is marked if there is some BK-basis of the restriction which can be extended to a BK-basis of $(C, A)$ :

Definition 13 [5] Let $S \subset \mathbb{C}^{n}$ be a $(C, A)$-invariant subspace. $S$ is said to be $(C, A)$-marked if there exists an adapted basis to $S$ in which the matrix of the pair $(C, A)$ has the form

$$
\left(\begin{array}{c}
\bar{A} \\
\bar{C}
\end{array}\right)=\left(\begin{array}{cc}
A_{1} & A_{3} \\
0 & A_{2} \\
\hline C_{1}^{\prime} & C_{3}^{\prime} \\
0 & C_{2}^{\prime}
\end{array}\right),
$$

and

(i) $\left(C_{1}^{\prime}, A_{1}\right)$ is a BK-matrix.

(ii) $(\bar{C}, \bar{A})$ is a BK-matrix, except for permutations, that is, there exists a permutation matrix $P \in M_{N}(\mathbb{C})$ such that $\left(\bar{C} P, P^{t} \bar{A} P\right)$ is a BK-matrix. 
Then we say that $(\bar{C}, \bar{A})$ is a marked matrix (with regard to $S$ ).

We will solve the equations in Theorem 11 explicitly in those cases where $\mathcal{A} \in \mathcal{M}^{*}$ is an observable marked matrix (then $r^{*}=m$ ). It can be easily observed (see [5] for further explanation) that if $\mathcal{A} \in \mathcal{M}^{*}$ is observable, $\mathcal{A} \in \mathcal{M}^{*}$ is marked if and only if there exists a matrix $\mathcal{A}_{c} \in \mathcal{O}_{\mathcal{A}}$ of the form described in the following definition which we will call its canonical form.

\section{Definition 14 Let}

$$
\mathcal{A}_{c}=\left(\begin{array}{cc}
A_{1} & A_{3} \\
0 & A_{2} \\
\hline C_{1} & 0
\end{array}\right) \in \mathcal{M}^{*}
$$

be an observable marked matrix with $q=\left(q_{1}, \ldots, q_{s}\right)$ and $p=\left(p_{1}, \ldots, p_{s}\right)$ being the BK-indices of the pairs $\left(C_{1}, A_{1}\right)$ and $(C, A)$, respectively, verifying $q_{1} \geq q_{2} \geq$ $\cdots \geq q_{s}, p_{i} \geq q_{i}, q_{1}+\cdots+q_{s}=d, p_{1}+\cdots+p_{s}=n$, and let us define $\delta_{i}=p_{i}-q_{i}$. Notice that $\left(p_{1}, \ldots, p_{s}\right)$ are not necessarily in non increasing order.

(i) We say that $\mathcal{A}$ is in canonical form (and then we write $\mathcal{A}_{c}$ ) if

(1) $\left(C_{1}, A_{1}\right)$ is a Brunovsky pair.

(2) $A_{2}=\operatorname{diag}\left(N_{\delta_{1}}, \ldots, N_{\delta_{s}}\right) \in M_{n-d}(\mathbb{C})$, where only $\delta_{i} \neq 0$ are considered.

(3) $A_{3}=\left(A_{3, i, j}\right)_{1 \leq i, j \leq s}, A_{3} \in M_{d, n-d}(\mathbb{C}), A_{3, i, j} \in M_{q_{i}, \delta_{j}}(\mathbb{C})$

$$
A_{3, i, i}=\left[\begin{array}{c}
e_{\delta_{i}} \\
0
\end{array}\right] \text { if } 1 \leq i \leq s, A_{3, i, j}=0 \text { otherwise, where only } \delta_{i} \neq 0
$$
are considered, and then $e_{\delta_{i}}$ will be a row matrix of zeros and one 1 in the position $\delta_{i}$ with the size corresponding to the context.

(ii) We say that all the matrices equivalent to the preceding one are of type $(q, p)$.

Example 1 The following matrix is an observable marked matrix in canonical form of type $((3,3,2,1),(4,3,6,3))$ : 


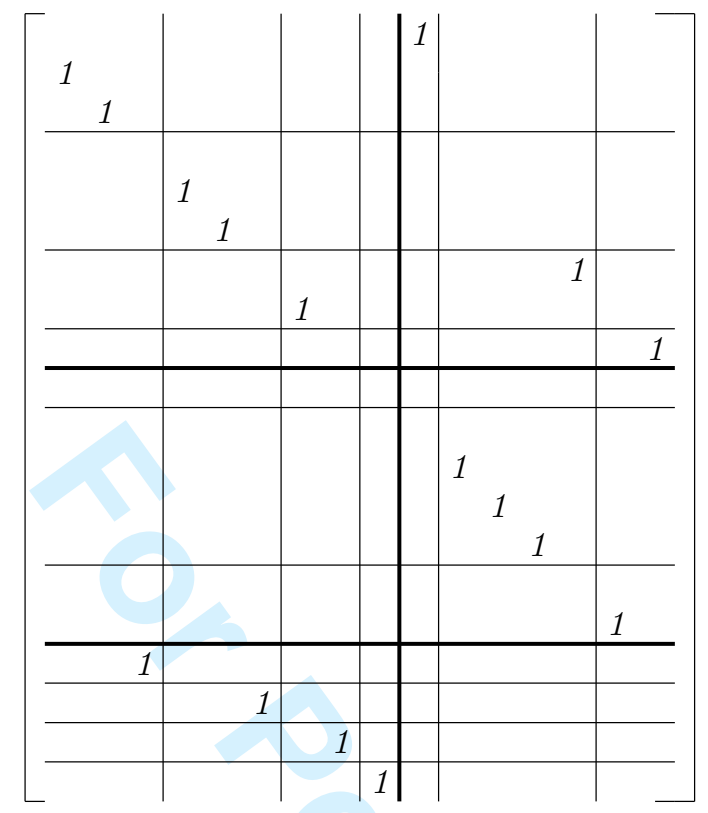

When we solve the set of equations in Theorem 12, the following special types of Toeplitz matrices often appear:

Definition 15 (1) We say that a matrix $X=\left(x_{i, j}\right) \in M_{\gamma, \beta}(\mathbb{C})$ is a Toeplitz matrix if it is constant along the diagonals.

(2) If $X$ is a Toeplitz matrix such that $x_{i, 1}=0$ if $i>1$, we say that $X$ is an upper Toeplitz matrix.

(3) We say that a block matrix $X=\left[X_{i, j}\right]_{1 \leq i \leq r, 1 \leq j \leq s}, \quad X_{i, j} \in M_{\gamma_{i}, \beta_{j}}(\mathbb{C})$ is a block Toeplitz matrix if each block $X_{i, j}$ is a T-matrix. We define a block upper Toeplitz matrix analogously.

We now solve equations (1) - (6) in Theorem 12 when the matrix $\mathcal{A}_{c} \in \mathcal{M}^{*}$ is an observable marked matrix in canonical form. The following theorem describes the corresponding solutions:

Theorem 16 (First Miniversal Deformation) Let $\mathcal{A}_{c} \in \mathcal{M}^{*}$ be an observable marked matrix in canonical form of type $(q, p)$ as in Definition 14.

Then, a miniversal deformation of $\mathcal{A}_{c} \in \mathcal{M}^{*}$ is given by the set of matrices

$$
\mathcal{A}_{c}+\left(\begin{array}{cc}
X_{1} & X_{3} \\
0 & X_{2} \\
\hline Y_{1} & Y_{2}
\end{array}\right)
$$

such that 
(2') $\left(Y_{1, h, k}\right)_{q_{k}}=0$,

(3') $\left(X_{1, h, k}\right)_{q_{k}}=0$,

(4') $\left[\begin{array}{c}X_{3, h, k} \\ Y_{2, h, k}\end{array}\right]$ UT-matrix,

$\left(5^{\prime}\right)\left[\begin{array}{cc}\left(X_{3, h, k}\right)_{\delta_{k}} & X_{1, h, k} \\ 0 & Y_{1, h, k}\end{array}\right]$ T-matrix if $\delta_{k}>0$,

$\left[\begin{array}{c}X_{1, h, k} \\ Y_{1, h, k}\end{array}\right]$ UT-matrix if $\delta_{k}=0$

(6') $\left[\begin{array}{c}X_{2, h, k} \\ \left(X_{3, h, k}\right)^{1}\end{array}\right]$ UT-matrix provided that $\delta_{h}>0$ and $\delta_{k}>0$,

where $(\cdot)_{\nu},(\cdot)^{\nu}$ mean, respectively, the $\nu$ column/row of $(\cdot)$.

\section{Proof.}

We will denote by $(\cdot)_{\widehat{\nu}}$ and $(\cdot)^{\widehat{\nu}}$ the matrix $(\cdot)$ from which the $\nu$ column/row, respectively, has been removed.

Solving each equation in Theorem 12, we have:

(1) $X_{2} C_{2}^{*}+Z=0$

Using that $C_{2}=0$ (from Definition 14), it turns out that $Z=0$.

(2) $Y_{1} C_{1}^{*}+Y_{2} C_{2}^{*}=0$

Also using that $C_{2}=0$ and the decomposition into blocks of the matrices, we have

$$
0=\sum_{i=1}^{m} Y_{1, h, i} C_{1, k, i}^{*}, \quad 1 \leq h, k \leq m
$$

and using the form of the blocks of $C_{1}$, we obtain $0=Y_{1, h, k} C_{1, k, k}^{*}$, Finally, using the introduced notation, this equation is equivalent $\left(Y_{1, h, k}\right)_{q_{k}}=0$.

(3) $X_{1} C_{1}^{*}+X_{3} C_{2}^{*}=0$

This equation is the same as (2), but the matrices involved are now $X_{1} \in$ $M_{d}(\mathbb{C}), X_{3} \in M_{d, n-d}(\mathbb{C})$.

(4) $X_{3} A_{2}^{*}-A_{1}^{*} X_{3}-C_{1}^{*} Y_{2}=0$

Using the decomposition into blocks of the matrices, we have

$$
\sum_{i=1}^{n} X_{3, h, i} A_{2, k, i}^{*}-\sum_{j=1}^{m} A_{1, j, h}^{*} X_{3, j, k}-\sum_{\ell=1}^{n} C_{1, \ell, h}^{*} Y_{2, \ell, k}=0,1 \leq h, k \leq m .
$$


Considering the form of the matrices $A_{1}, A_{2}$ and $C_{1}$, we have $X_{3, h, k} N_{\delta_{k}}^{*}-$ $N_{q_{h}}^{*} X_{3, h, k}-e_{q_{h}}^{*} Y_{2, h, k}=0$; and using the above notation, we obtain $\left[\begin{array}{cc}0 & \left(X_{3, h, k}\right)_{\widehat{\delta_{k}}}\end{array}\right]=$ $\left[\begin{array}{c}\left(X_{3, h, k}\right)^{\hat{1}} \\ Y_{2, h, k}\end{array}\right]$, which is equivalent to $\left(4^{\prime}\right)$.

(5) $X_{1} A_{1}^{*}+X_{3} A_{3}^{*}-A_{1}^{*} X_{1}-C_{1}^{*} Y_{1}=0$

Using the decomposition into blocks, the last equation is equal to

$$
\sum_{i=1}^{m} X_{1, h, i} A_{1, k, i}^{*}+\sum_{i=1}^{n} X_{3, h, i} A_{3, k, i}^{*}=\sum_{i=1}^{m} A_{1, i, h}^{*} X_{1, i, k}+\sum_{i=1}^{n} C_{1, i, h}^{*} Y_{1, i, k}, 1 \leq h, k \leq m .
$$

Considering the form of the matrices, we distinguish two cases:

(a) If $\delta_{k}>0, \quad X_{1, h, k} N_{q_{k}}^{*}+X_{3, h, k}\left[\begin{array}{c}e_{\delta_{k}} \\ 0\end{array}\right]^{*}=N_{q_{h}}^{*} X_{1, h, k}+e_{q_{h}}^{*} Y_{1, h, k}$.

Using the above notation, we have $\left[\left(X_{3, h, k}\right)_{\delta_{k}},\left(X_{1, h, k}\right)_{\widehat{q_{k}}}\right]=\left[\begin{array}{c}\left(X_{1, h, k}\right)^{\widehat{1}} \\ Y_{1, h, k}\end{array}\right]$, which is equivalent to $\left(5^{\prime}\right)$.

(b) If $\delta_{k}=0, \quad X_{1, h, k} N_{q_{k}}^{*}=N_{q_{h}}^{*} X_{1, h, k}+e_{q_{h}}^{*} Y_{1, h, k}$.

Using the above notation, we have $\left[0,\left(X_{1, h, k}\right)_{\widehat{q_{k}}}\right]=\left[\begin{array}{c}\left(X_{1, h, k}\right)^{\widehat{1}} \\ Y_{1, h, k}\end{array}\right]$.

Therefore, the solution for this case is equivalent to considering $\left(X_{3, h, k}\right)_{\delta_{k}}=$ 0 in the general case, and this gives us (5').

(6) $X_{2} A_{2}^{*}-A_{3}^{*} X_{3}-A_{2}^{*} X_{2}-C_{2}^{*} Y_{2}=0$

Using the decomposition into blocks of the matrices and that $C_{2}=0$, we have

$\sum_{i=1}^{n} X_{2, h, i} A_{2, k, i}^{*}-\sum_{i=1}^{m} A_{3, i, h}^{*} X_{3, i, k}-\sum_{i=1}^{n} A_{2, i, h}^{*} X_{2, i, k}=0,1 \leq h, k \leq$ $m, \quad \delta_{h}>0, \delta_{k}>0$.

Considering the form of the matrices, we have $X_{2, h, k} N_{\delta_{k}}^{*}-A_{3, h, h}^{*} X_{3, h, k}-$ $N_{\delta_{h}}^{*} X_{2, h, k}=0$. This equation is equivalent to $\left[0,\left(X_{2, h, k}\right)_{\widehat{\delta_{k}}}\right]=\left[\begin{array}{l}\left(X_{2, h, k}\right)^{\widehat{1}} \\ \left(X_{3, h, k}\right)^{1}\end{array}\right]$, and we obtain $\left(6^{\prime}\right)$.

Example 2 Given the observable marked matrix in canonical form of type $((3,3,2,1),(4,3,6,3))$ in Example 1, its miniversal deformation given by Theorem 16 is 


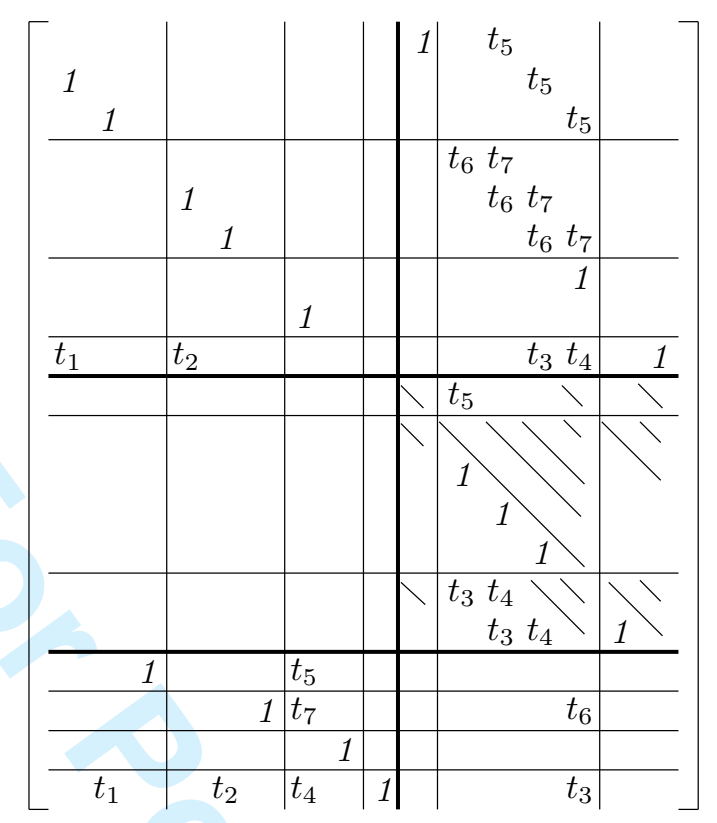

where the parameters shown with the lines appear only in the corresponding block and the elements on each line are the same, and $t_{i}$ are the parameters appearing in more than one block in $X_{1}, X_{2}, X_{3}, Y_{1}, Y_{2}$.

As an application we will compute $\operatorname{codim} \mathcal{O}_{\mathcal{A}_{c}}$ in Section 5. In fact, we use it to derive a new miniversal deformation of $\mathcal{A}_{c} \in \mathcal{M}^{*}$ without repeated parameters, which will be more useful to study the effect of each parameter.

Definition 17 Let $\mathcal{A}_{c} \in \mathcal{M}^{*}$ be an observable marked matrix in canonical form (see Definition 14). We define the matrices $\mathcal{C}_{1 h k}^{i}, \mathcal{A}_{2 h k}^{i}$ and $\mathcal{A}_{3 h k}^{i}$ in $M_{m, n}(\mathbb{C}) \times$ $M_{n}(\mathbb{C})$ having the same block sizes as $\mathcal{A}_{c} \in \mathcal{M}^{*}$, and all the entries 0 except one 1 placed in the first row of the block $C_{1 h k}, A_{2 h k}$ or $A_{3 h k}$, respectively, and in their i-column.

We will be mainly interested in the following subspaces spanned by some of these matrices:

1. $\mathcal{S}_{1}=\left[\mathcal{C}_{1 h k}^{l}: \quad 1 \leq h, k \leq m, \quad q_{h}<l \leq q_{k}-1\right]$. We will write it

$$
\left(\begin{array}{cc}
0 & 0 \\
0 & 0 \\
\hline Y_{1}^{\text {obs }} & 0
\end{array}\right),
$$

$\left(C_{1}+Y_{1}^{\text {obs }}, A_{1}\right)$ is the miniversal deformation in [12] of the observable pair $\left(C_{1}, A_{1}\right)$, 
2. $\mathcal{S}_{2}=\left[\mathcal{A}_{2 h k}^{i}: \quad 1 \leq h, k \leq m, \quad \delta_{k}-\delta_{h}<i \leq \delta_{k}\right]$. We will write it

$$
\left(\begin{array}{cc}
0 & 0 \\
0 & X_{2}^{\text {end }} \\
\hline 0 & 0
\end{array}\right),
$$

$A_{2}+X_{2}^{e n d}$ is the miniversal deformation in [1] of the square matrix $A_{2}$.

3. $\mathcal{S}_{3}$ spanned by the matrices $\mathcal{A}_{3 h k}^{j}$ with $1 \leq h, k \leq m$, and the index $j$ varying as follows:

if $\delta_{h}>0, \quad p_{h}-q_{h}<j \leq \delta_{k}-q_{h}$, and $\max \left(0, p_{h}-\delta_{k}\right)+\delta_{k}-q_{h}<j \leq$ $\min \left(q_{h}, q_{k}-1\right)+\delta_{k}-q_{h}$;

if $\left.\delta_{h}=0, \quad 0<j \leq \min \left(q_{k}-1, p_{k}-q_{h}-1, \delta_{k}\right)+\delta_{k}-q_{h}\right]$. We will write it

$$
\left(\begin{array}{cc}
0 & X_{3}^{\prime} \\
0 & 0 \\
\hline 0 & 0
\end{array}\right) \text {. }
$$

Theorem 18 (Second Miniversal Deformation) Let $\mathcal{A}_{c} \in \mathcal{M}^{*}$ be an observable marked matrix in canonical form of type $(q, p)$ as in Definition 14 and $S_{\mathcal{A}_{c}}=\mathcal{S}_{1}+\mathcal{S}_{2}+\mathcal{S}_{3}$ (see Definition 17).

Then, a miniversal deformation of $\mathcal{A}_{c} \in \mathcal{M}^{*}$ is given by the subvariety $\mathcal{A}_{c}+$ $S_{\mathcal{A}_{c}}$, that is to say, it is given by the set of matrices

$$
\mathcal{A}_{c}+\left(\begin{array}{cc}
0 & X_{3}^{\prime} \\
0 & X_{2}^{\text {end }} \\
\hline Y_{1}^{\text {obs }} & 0
\end{array}\right) \text {. }
$$

\section{Proof.}

By construction, the generators of $S_{\mathcal{A}_{c}}$ are linearly independent and its dimension is the codimension of $\mathcal{O}_{\mathcal{A}_{c}}$. We will see that $S_{\mathcal{A}_{c}}$ is a supplementary subspace of $T_{\mathcal{A}_{c}} \mathcal{O}_{\mathcal{A}_{c}}$ by proving that its intersection is the null space. In order to do so, we consider the usual scalar product in matrix spaces $\langle M, N\rangle=\operatorname{trace}\left(M N^{*}\right)$, where $N^{*}$ means the conjugate-transpose matrix of $N$, and we will prove that for every non null vector of $S_{\mathcal{A}_{c}}$, there is a vector of $\left(T_{\mathcal{A}_{c}} \mathcal{O}_{\mathcal{A}_{c}}\right)^{\perp}$ such that their product is not zero.

Notice that if $\mathcal{X} \in\left(T_{\mathcal{A}_{c}} \mathcal{O}_{\mathcal{A}_{c}}\right)^{\perp}$, we then have

$$
\begin{aligned}
& <\mathcal{X}, \mathcal{A}_{2 h k}^{i}>=\left(X_{2 h k}\right)_{1, i}, \\
& <\mathcal{X}, \mathcal{A}_{3 h k}^{j}>=\left(X_{3 h k}\right)_{1, j}, \\
& <\mathcal{X}, \mathcal{C}_{1 h k}^{l}>=\left(Y_{1 h k}\right)_{l} .
\end{aligned}
$$


Now let

$$
\mathcal{V}=\sum_{h, k, i} x_{2 h k}^{i} \mathcal{A}_{2 h k}^{i}+\sum_{h, k, j} x_{3 h k}^{j} \mathcal{A}_{3 h k}^{j}+\sum_{h, k, l} y_{1 h k}^{l} \mathcal{C}_{1 h k}^{l}
$$

be an element of $S_{\mathcal{A}_{c}}$.

We consider the element $\mathcal{X}=\left(\begin{array}{cc}X_{1} & X_{3} \\ 0 & X_{2} \\ \hline Y_{1} & Y_{2}\end{array}\right) \in\left(T_{\mathcal{A}_{c}} \mathcal{O}_{\mathcal{A}_{c}}\right)^{\perp}$ defined by

$$
\begin{aligned}
\left(X_{2 h k}\right)_{1, i} & =\overline{x_{2 h k}^{i}}, \\
\left(X_{3 h k}\right)_{j} & =\overline{x_{3 h k}^{j}}, \\
\left(Y_{1 h k}\right)_{l} & =\overline{y_{1 h k}^{l}}
\end{aligned}
$$

where the indices vary as in Definition 17.

Then,

$$
<\mathcal{V}, \mathcal{X}>=\sum_{h, k, i}\left|x_{2 h k}^{i}\right|^{2}+\sum_{h, k, j}\left|x_{3 h k}^{j}\right|^{2}+\sum_{h, k, l}\left|y_{1 h k}^{l}\right|^{2}
$$

and this implies that $\langle\mathcal{V}, \mathcal{X}\rangle=0$ if and only if $\mathcal{V}=0$.

Hence, we have proved that $S_{\mathcal{A}_{c}}$ is a supplementary subspace of $T_{\mathcal{A}_{c}} \mathcal{O}_{\mathcal{A}_{c}}$.

Example 3 The new miniversal deformation in Example 2 is

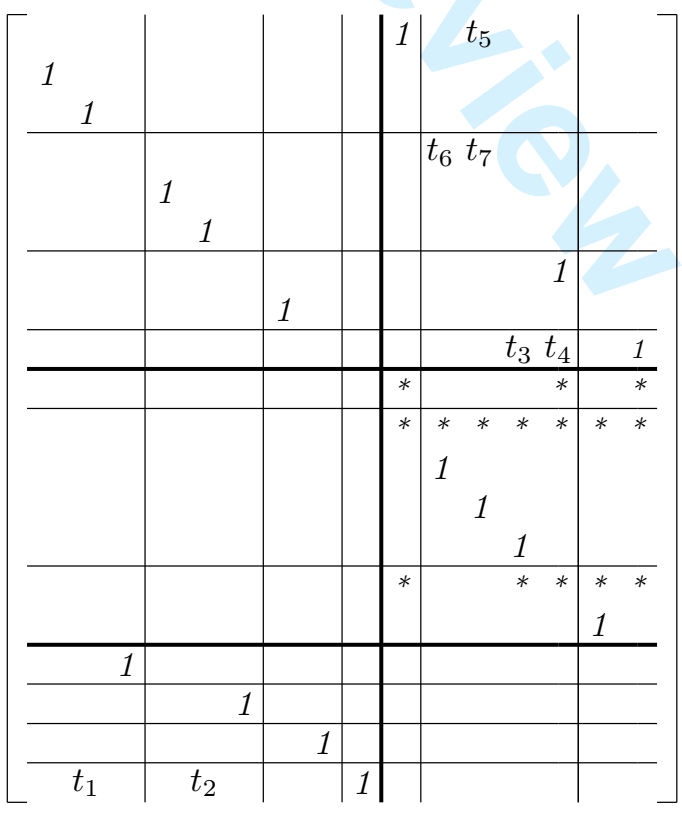




\section{Applications}

\subsection{Dimension of the orbit}

As an application of Theorem 16, we obtain the dimension of $\mathcal{O}_{\mathcal{A}_{c}}$.

Corollary 19 Let $\mathcal{A}_{c} \in \mathcal{M}^{*}$ be an observable marked matrix in canonical form of type $(q, p)$ as in Definition 14. Then, the codimension of its orbit is

$$
\begin{aligned}
\operatorname{codim} \mathcal{O}_{\mathcal{A}_{c}} & =\sum_{\substack{1 \leq h, k \leq n \\
\delta_{h} \cdot \delta_{k}>0}} \min \left(\delta_{h}, \delta_{k}\right)+\sum_{\substack{1 \leq h, k \leq n \\
\delta_{h} \cdot \delta_{k}>0}} \max \left(0, \min \left(q_{h}, q_{k}-1\right)-\max \left(0, p_{h}-\delta_{k}\right)\right) \\
& +\sum_{\substack{1 \leq h, k \leq n \\
\delta_{h} \cdot \delta_{k}>0}} \max \left(0, \delta_{k}-p_{h}\right)+\sum_{1 \leq h, k \leq n} \max \left(0, q_{k}-q_{h}-1\right) \\
& +\sum_{\substack{1 \leq h, k \leq n \\
\delta_{h}=0}} \min \left(q_{k}-1, p_{k}-q_{h}-1, \delta_{k}\right)+\sum_{\substack{1 \leq h, k \leq n \\
\delta_{h}=0}} \max \left(0, \delta_{k}-q_{h}\right) .
\end{aligned}
$$

\section{Proof.}

To count how many freedom degrees the miniversal deformation has, we study the number of parameters appearing in the solution $\mathcal{X}=\left(\begin{array}{cc}X_{1} & X_{3} \\ 0 & X_{2} \\ \hline Y_{1} & Y_{2}\end{array}\right)$. The following figure is useful because it shows all the nullity and constancy conditions of the diagonals appearing in Theorem 16:

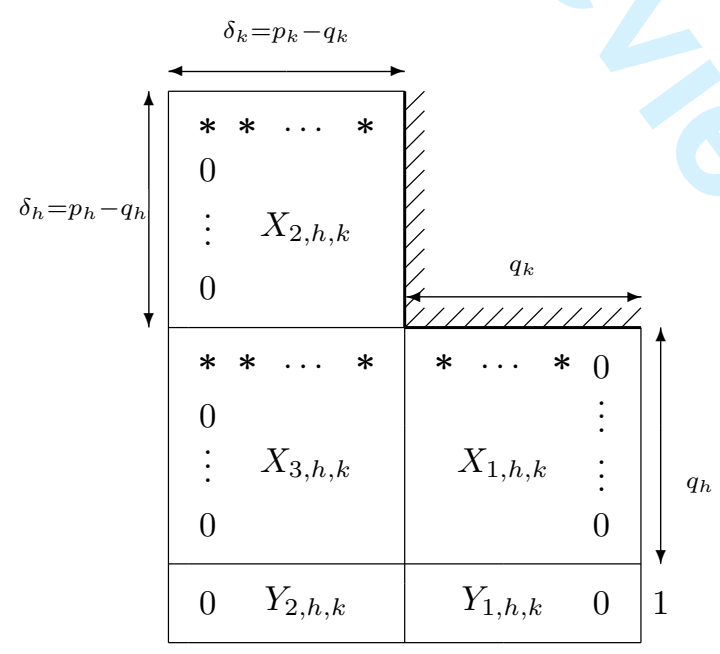

We denote by $*$ the origin of the diagonals that can be different from zero. The remaining conditions of Theorem 16 and the sizes of the blocks will allow us to obtain the actual ones. 
We distinguish three different cases depending on the number of blocks in the above figure:

(I) $\delta_{h}, \delta_{k}>0$

In this case, all the blocks of the solution appear as the above figure shows. It can be seen that there are four types of parameters:

(a) Those beginning and finishing in $X_{2, h, k}$.

(b) Those beginning in $X_{2, h, k}$ and finishing in $Y_{1, h, k}$.

(c) Those beginning in $X_{2, h, k}$ and finishing in $Y_{2, h, k}$.

(d) Those beginning in $X_{1, h, k}$ and finishing in $Y_{1, h, k}$.

Indeed, $\left(X_{2, h, k}\right)_{1, i}=\left(X_{2, h, k}\right)_{1-i+\delta_{k}, \delta_{k}} \quad 1 \leq i \leq \delta_{k}$. We distinguish the cases:

(a) If $\delta_{k}-\delta_{h}<i \leq \delta_{k}$.

(b) If $0<i \leq \delta_{k}, \delta_{k}<i+p_{h}<p_{k}, \quad\left(X_{2, h, k}\right)_{1, i}=\left(Y_{1, h, k}\right)_{i+p_{h}}$ and $i+\delta_{h} \leq$ $\delta_{k}$ because $\left(Y_{1, h, k}\right)_{q_{k}}=0$.

(c) If $p_{h}<1-i+\delta_{k}, \quad\left(X_{2, h, k}\right)_{1, i}=\left(Y_{2, h, k}\right)_{i+p_{h}}$.

(d) $\left(X_{1, h, k}\right)_{1, j}=\left(Y_{1, h, k}\right)_{j+q_{h}} \quad 1 \leq j \leq q_{k}$, and $j+q_{h}<q_{k}$ because $\left(Y_{1, h, k}\right)_{q_{k}}=0$.

In summary, and taking as reference the elements of the first row of $X_{2, h, k}$ in case (a) and the elements of the blocks of $Y$ in the other ones, we have the following parameters:

(a) $\left(X_{2, h, k}\right)_{1, i}$ for $\quad \delta_{k}-\delta_{h}<i \leq \delta_{k}$.

(b) $\left(Y_{1, h, k}\right)_{j}$ for $\max \left(0, p_{h}-\delta_{k}\right)<j \leq \min \left(q_{h}, q_{k}-1\right)$.

(c) $\left(Y_{2, h, k}\right)_{j}$ for $p_{h}<j \leq \delta_{k}$.

(d) $\left(Y_{1, h, k}\right)_{i}$ for $q_{h}<i \leq q_{k}-1$.

Adding up these four types of parameters, classified according to whether they finish in $X_{2, h, k}, Y_{2, h, k}$ or $Y_{1, h, k}$, respectively, for the case $\delta_{h}, \delta_{k}>0$ we obtain the following total number of freedom degrees:

$$
\begin{gathered}
\min \left(\delta_{h}, \delta_{k}\right)+\max \left(0, \min \left(q_{h}, q_{k}-1\right)-\max \left(0, p_{h}-\delta_{k}\right)\right)+\max \left(0, \delta_{k}-p_{h}\right) \\
+\max \left(0, q_{k}-q_{h}-1\right) .
\end{gathered}
$$

(II) $\delta_{k}=0$

In this case, we only have the blocks $X_{1, h, k}$ and $Y_{1, h, k}$. Repeating the reasoning of (I), if $\delta_{k}=0$, there only exist the parameters beginning in $X_{1, h, k}$, that is, those given by (d), and the number of parameters is

$$
\max \left(0, q_{k}-q_{h}-1\right) .
$$


(III) $\delta_{h}=0, \delta_{k}>0$

In this case, we only have the blocks $X_{1, h, k}, X_{3, h, k}, Y_{1, h, k}$ and $Y_{2, h, k}$. It can be seen that there are three types of parameters:

(d) Those beginning in $X_{1, h, k}$ and finishing in $Y_{1, h, k}$.

(e) Those beginning in $X_{3, h, k}$ and finishing in $Y_{1, h, k}$.

(f) Those beginning in $X_{3, h, k}$ and finishing in $Y_{2, h, k}$.

Adding up these three types of parameters, for the case $\delta_{h}=0, \delta_{k}>0$ we obtain the following total number of freedom degrees:

$$
\max \left(0, q_{k}-q_{h}-1\right)+\min \left(q_{k}-1, p_{k}-q_{h}-1, \delta_{k}\right)+\max \left(0, \delta_{k}-q_{h}\right) .
$$

Adding up all the preceding cases and grouping them according to the block of the reference parameter, we have the formula of the corollary.

Example 4 For the observable marked matrix in Example 1, the addends in Corollary 19 give, respectively, 15,2,1,2,1 and 1, which correspond to the parameters $\left(^{*}\right),\left(t_{4}, t_{5}\right),\left(t_{3}\right),\left(t_{1}, t_{2}\right),\left(t_{7}\right)$ and $\left(t_{6}\right)$.

\section{$5.2 \quad$ Structural stability}

Now we study the observable marked matrices with open orbit, that is, the ones with the dimension of the manifold as the dimension of their orbit. They are known as structurally stable matrices.

Definition 20 [23] Let $\mathcal{M}$ be a topological manifold, when an equivalence relation is defined. A point $x \in \mathcal{M}$ is said to be structurally stable if its equivalence class contains an open neighborhood of $x$.

We see that there are no non-trivial pairs of this type.

Corollary 21 The observable marked matrices $\mathcal{A} \in \mathcal{M}^{*}$ structurally stable with regard to the equivalence relation in Definition 5 are only those of the trivial case:

(i) $d=n$,

(ii) $\left|p_{i}-p_{j}\right| \leq 1$.

\section{Proof.}

Let $\mathcal{A} \in \mathcal{M}^{*}$ be an observable marked matrix in canonical form of type $(q, p)$. Then, it will be structurally stable if and only if its miniversal deformation is null. Or, equivalently, if codim $\mathcal{O}_{\mathcal{A}}=0$. This means that each term in Corollary 19 
must be 0 . From the first one, it follows $\delta_{i}=0$, for all $i \leq m$, and then $d=n$. Now the fourth term gives us $\left|p_{k}-p_{h}\right| \leq 1$ for $1 \leq h, k \leq s$.

Conversely, all the terms are null if (i)-(ii) hold.

Notice that condition (i) implies $\mathcal{M}^{*}=M_{n+m, n}(\mathbb{C})$, that is, $\mathcal{A} \in \mathcal{M}^{*}$ is a full rank observable pair. Then, condition (ii) is exactly that obtained in [12] for this particular case.

\subsection{Bifurcation diagrams}

The simplicity of the miniversal deformation in Theorem 18 allows us to study the effect of each parameter in the invariants of $\mathcal{A}_{c}$. It is clear that the Jordan form of $A_{2}$ is perturbed by $X_{2}^{\text {end }}$ just in Arnold's works. Let see the effect of $Y_{1}^{\text {obs }}$ and $X_{3}^{\prime}$.

As we have pointed out, $\left(C_{1}+Y_{1}^{\text {obs }}, A_{1}\right)$ is the miniversal deformation in [12] of the restriction $\left(C_{1}, A_{1}\right)$. Hence, its effect is the well-known perturbation of the BK-indices of an observable pair: the parameter corresponding to $\mathcal{C}_{1 h k}^{l}$ transforms $q_{k}$ into 1 , and $q_{h}$ into $q_{h}+q_{k}-l$. In general, the initial BK-indices $q_{1}, \ldots, q_{s}$ will change into new indices $q_{1}^{\prime}, \ldots, q_{s}^{\prime}$ such that they are majorized by the initial ones in the following sense (see [19]):

$q_{1}^{\prime} \leq q_{1} ; \quad q_{1}^{\prime}+q_{2}^{\prime} \leq q_{1}+q_{2} ; \quad \cdots ; \quad q_{1}^{\prime}+\cdots+q_{s-1}^{\prime} \leq q_{1}+\cdots+q_{s-1} ; \quad q_{1}^{\prime}+\cdots+q_{s}^{\prime}=$ $q_{1}+\cdots+q_{s}$.

That is, the BK-indices of the restriction will be balanced until the maximal difference between them is 1 (or 0 ).

In Example 3, for instance, if some of the parameters $t_{1}, t_{2}$ in $Y_{1}^{\prime}$ are non zero, then $\left(q_{1}^{\prime}, q_{2}^{\prime}, q_{3}^{\prime}, q_{4}^{\prime}\right)=(3,2,2,2)$.

Note that they are the only BK-indices compatible with the above majorization relations and are structurally stable because the maximal difference between them is 1 .

Similarly, the BK-indices of $(C, A)$ will be perturbed into majorized ones as above. For instance, in Example 3 the only BK-indices majorized by the initial ones $(6,4,3,3)$ are $(5,5,3,3),(5,4,4,3)$ and $(4,4,4,4)$. In fact, the bifurcation diagram in $X_{3}^{\prime}$ is

$(6,4,3,3) \quad$ if $\quad t_{3}=t_{4}=t_{5}=t_{6}=t_{7}=0$,

$(5,5,3,3) \quad$ if $\quad t_{5} \neq 0, \quad t_{3}=t_{4}=t_{6}=t_{7}=0$,

$(5,4,4,3)$ if $t_{4} t_{6}-t_{3} t_{7}=0, \quad$ some of them being non zero,

$(4,4,4,4) \quad$ if $\quad t_{4} t_{6}-t_{3} t_{7} \neq 0$.

\section{References}

[1] V.I. Arnold, On Matrices Depending on Parameters, Uspekhi Mat. Nauk., 26 (1971), p. 101-114. 
[2] V.I. Arnold, Arnold's Problems, Springer, Berlin (2004).

[3] G. Basile; G. Marro, Controlled and conditioned invariant subspaces in linear system theory, J. Optim. Theory Appl., 3 (1969), p. 306-315.

[4] Th. Brocker; L. Lander, Differentiable Germs and Catastrophes, Cambridge University Press, Cambridge (1975).

[5] A. Compta; J. Ferrer, On $(A, B)^{t}$-invariant Subspaces Having Extendible Brunovsky Bases, Linear Algebra Appl., 225 (1997), p. 185-201.

[6] A. Compta; J. Ferrer, A Geometric Approach to the Carlson Problem, SIAM Journal on Matrix Analysis Appl. Vol. 22, n. 1(2000), p. 258-275.

[7] A. Compta; J. Ferrer, Matricial Realizations of the Solutions of the Carlson Problem, Linear Algebra Appl., 353 (2002), p. 197-206.

[8] A. Compta; J. Ferrer; M. Peña, Dimension of the Orbit of Marked Subspaces,Linear Algebra Appl., 379 (2004), p. 239-248.

[9] A. Compta; J. Ferrer; M. PeñA, Perturbations preserving conditioned invariant subspaces, Mathematical Methods in the Applied Sciences, (2011), DOI: 10.1002/mma.1553.

[10] A. Compta; J. Ferrer; F. Puerta, Miniversal deformations of marked matrices, Linear Algebra Appl., 361 (2003), p. 181-201.

[11] A. Compta; U. Helmke; M. Peña; X. Puerta, Simultaneous Versal Deformations of Endomorphisms and Invariant Subspaces, Linear Algebra Appl., 413 (2006), p. 303-318.

[12] J. Ferrer; M.I. García; F. Puerta, Brunovsky Local Form of a Holomorphic Family of Pais of Matrices, Linear Algebra Appl., 253 (1997), p. 175-198.

[13] J. Ferrer; M.I. García; F. Puerta, Regularity of the BrunovskyKronecker Stratification, SIAM Journal on Matrix Analysis Appl. Vol. 21, n. 3 (2000), p. $724-742$.

[14] J. Ferrer; F. Puerta, Similarity of Non-everywhere Defined Linear Maps, Linear Algebra Appl., 150 (1992), p. 27-55.

[15] J. Ferrer; F. Puerta, Versal Deformations of Invariant Subspaces, Linear Algebra Appl., 332-334 (2001), p. 569-582.

[16] J. Ferrer; F. Puerta; X. Puerta, Differentiable Structure of the Set of Controllable $(A, B)^{t}$-invariant Subspaces, Linear Algebra Appl., 275-276 (1998), p. 161-177. 
[17] J. Ferrer; F. Puerta; X. Puerta, Stratification and Bundle Structure of the Set of Conditioned Invariant Subspaces in the General Case, Systems and Control Letters, 48 (2003), p. 77-86.

[18] I. Gohberg; P. Lancaster; L. Rodman, Invariant Subspaces of Matrices with Applications. Wiley, New York (1986).

[19] M. Gracia; I. De Hoyos; I. Zaballa, Perturbation of Linear Control Systems, Linear Algebra Appl., 121 (1989), p. 353-383.

[20] V. Guillemin; A. Pollack, Differential Topology. Prentice-Hall, New Jersey (1974).

[21] M.A. Shayman, On the Variety of Invariant Subspaces of a Finitedimensional Linear Operator, Trans. Amer. Math. Soc., vol. 274 (2) (1982), p. $721-747$.

[22] A. Tannenbaum, Invariance and System Theory: Algebraic and Geometric Aspects, LNM, n. 845, Springer (1981).

[23] J.C. Willems, Topological classification and structural stability of linear systems, J. Differential Equations, 35 (1980), p. 306-318.

[24] W.M. Wonham, Linear Multivariable Control: A Geometric Approach, Springer, New York (1979). 